\title{
Towards a Reference Architecture for Augmentative and Alternative Communication Systems
}

\author{
Natália M. Franco ${ }^{1}$, Edson A. Silva ${ }^{1}$, Rinaldo J. Lima ${ }^{2}$, Robson N. Fidalgo ${ }^{1}$ \\ ${ }^{1}$ Informatics Center - Federal University of Pernambuco (UFPE) \\ Recife - PE - Brazil \\ ${ }^{2}$ Department of Statistics and Informatics - Federal Rural University of Pernambuco (UFRPE) \\ Recife - PE - Brazil \\ $\{\mathrm{nmf}$, eas4,rdnf\}@cin.ufpe.br, rinaldo.jose@ufrpe.br
}

\begin{abstract}
Augmentative and Alternative Communication (AAC) systems are broadly used to expand communication skills of individuals with severe communicative disorders. These systems should prioritize requirements that contribute to making the communication easy and effective to be robust. However, there is no guideline available to aid the implementation of these systems. In this paper, we analyze the fragmented knowledge available on scientific proposals and technological solutions to extract key concepts for a robust AAC system. Then, we propose a Reference Architecture (RA) that can be used as a conceptual guide to define and evaluate concrete and robust software architectures for several goals including literacy in inclusive education.
\end{abstract}

\section{Introduction}

Augmentative and Alternative Communication (AAC) attempts to compensate difficulties or disabilities demonstrated by individuals with severe communicative disorders (e.g., autism, cerebral palsy and dysarthria). AAC systems are broadly used for functional communication and for expanding communication skills because these are based on pictograms (i.e., figurative drawings - cf. Figure 1-A) that can have a label, and represent concepts (e.g., object, actions, and feelings) alone or in association with other pictograms. Besides that, AAC systems are also used for educational activities in inclusive education such as introducing the symbolic communication (i.e., by teaching how to use pictograms to communicate), improving language skills (i.e., by making communication faster), and facilitating the literacy process (i.e., by teaching syntactically and semantically correct complements). Indeed, empirical and theoretical evidence found in [Kagohara et al. 2013], [Lima et al. 2017], [Franco et al. 2017] shows that the AAC system adoption returned good results in inclusive education.

Despite the importance of AAC and the number of systems available (cf. Section 2 ), we highlight that the knowledge produced is fragmented into several scientific proposals and technological solutions, consequently there is no consolidated baseline for developing robust AAC systems. To overcome these shortcomings, a Reference Architecture (RA) can be used as a conceptual guide that compiles key concepts, relationships, and features of a domain to define a template solution for a concrete software architecture [Cloutier et al. 2010]. That is, an RA specifies an abstract and 
VII Congresso Brasileiro de Informática na Educação (CBIE 2018)

Anais do XXIX Simpósio Brasileiro de Informática na Educação (SBIE 2018)

agnostic solution that guides and constrains the instantiations of concrete software architectures, ensuring the standardization and interoperability, as well as, reducing costs/risks and increasing the quality of these instantiations. To the best of our knowledge (cf. Section 2), there are no concurrent proposals of an RA for AAC. For this reason, in this paper we present the first effort towards an RA for developing and evaluating AAC systems robust enough to effective usage in communication and education of disabled people.

\section{Existing AAC Systems}

We did exhaustive research by "Reference Architecture" AND "Augmentative and Alternative Communication" in the main digital libraries (i.e., IEEE, ACM, Scopus, Springer, Science Direct and Elsevier), but no article was found. However, some authors have already (i) proposed computational resources and software architectures for AAC systems; or (ii) analyzed and discussed challenges of AAC systems.

In the first group, we analyzed three articles. Pino and Kouroupetroglou (2010) proposed ITHACA, a framework for AAC that provides adaptable, modular and multilingual AAC products. ITHACA encompasses modules for symbols navigation and selection, and a syntactic parser that relies on pre-stored messages and also expands telegraphic input (e.g., "Daddy here") to well-formed and grammatically corrected sentences (e.g., "Daddy is here"). However, this syntactic parser does not support novel utterance generation. Hernández et al. (2014) developed a recommendation model using questions and answers aiming at reducing the time employed in the construction of coherent and semantically correct sentences. The user can start the sentence at any of its constituent parts and, in the end, the model corrects the order of the sentence and introduces extra sentence parts for a corrected sentence. This recommendation model does not support syntactic coherence nor vocabulary customization for each user. Martínez-Santiago et al. (2015) proposed the Simple Upper Ontology (SUpO), a language-independent semantic grammar for modeling the language of a beginner communicator. SUpO is rooted in both the semantic model of FrameNet ${ }^{1}$ and the syntactic model of Grammatical Framework ${ }^{2}$. The authors argue that an upper ontology for this context is feasible because the vocabulary of a beginner communicator is small and only used for straightforward assertions. However, SUpO has the drawback that it does not support the inclusion of new words into the first vocabulary.

In the second group, we analyzed three articles too. Kagohara et al. (2013) conducted a systematic review of studies that involved mobile devices in teaching programs for individuals with developmental disabilities and results suggest that these devices are viable technological aids for individuals with developmental disabilities. McNaughton and Light (2013) discuss the benefits of mobile technologies for AAC, including the greater functionality and interconnectivity and diffusion of AAC research and development. The authors also discuss challenges that an AAC system must address, including (i) the focus in the communication - not just technology -, (ii) the support of a variety of communicational functions, and (iii) the development of

\footnotetext{
${ }^{1}$ FrameNet - https://framenet.icsi.berkeley.edu/fndrupal/

${ }^{2}$ Grammatical Framework - https://www.grammaticalframework.org/
} 
VII Congresso Brasileiro de Informática na Educação (CBIE 2018)

Anais do XXIX Simpósio Brasileiro de Informática na Educação (SBIE 2018)

innovative approaches to AAC assessment. Franco et al. (2017) proposed a checklist to evaluate the functionalities of AAC systems as well as its adherence to the Nielsen's Heuristics that corresponds to general principles for the user interface.

\section{Requirements of a Robust AAC System}

AAC developers should prioritize some functional requirements for developing robust AAC systems [Kagohara et al. 2013], [McNaughton and Light 2013], [Newel et al. 1998]. These requirements include: 1) Vocabulary customization - the system must allow the selection and organization of the vocabulary, as well as its language representation method; 2) Interface customization - the system must allow different configurations for customizing the way the user see, listen and manipulate the interface; 3) Communication flexibility - the system must allow freedom for the user to say whatever they want, shortcuts to increase the communication speed; 4) Appropriate feedback - the system must provide an understanding of its status for the user through visual and sound notifications; 5) Construction of meaningful sentences - the system must provide clues to help users to select pictograms and construct syntactic and semantically correct sentences. We grouped these requirements into two groups that we will detail next: 1) Customization (i.e., Vocabulary customization and Interface customization); and 2) Communication (i.e., Communication flexibility, Appropriate feedback and Construction of meaningful sentences).

\subsection{Customization}

Here we deal with two major aspects of customization: vocabulary and interface. The first is presented as follow, while the second is detailed in next paragraph. The vocabulary customization comprises (i) the vocabulary selection, (ii) the Language Representation Method (LRM) and (iii) the vocabulary organization. The vocabulary selection concerns the choice for a controlled and extensive vocabulary that allows effective user's communication in its daily communication needs. This controlled vocabulary can be divided into two main groups according to its usage: core and fringe vocabulary [Balandin and Iacono 1999]. A core vocabulary consists in a small set of words with two distinct properties: its high level of commonality (i.e., the words of the core vocabulary are shared among many users) and its high usage frequency by many users. In contrast, a fringe vocabulary is often extensive in the number of its constituent words, changes frequently, and is highly individualized (i.e., low level of commonality) among users. The importance of core words was proved by statistical analyses over different age groups [Balandin and Iacono 1999], [Franco et al. 2017]. Vocabulary selection should equally deal with the pragmatic functions of communication (e.g., requesting assistance or information, and responding to questions), the context in which the users are expected to participate (e.g., home, school, and therapy), and the communication development of them (e.g., cognitive level or Mean Length of Utterance $^{3}$ ). After the vocabulary selection, one needs to choose the Language Representation Method (LRM) [Hill 2006] among: (i) Alphabet-Based - i.e., one concept is defined by a word; (ii) Single Meaning Picture (SMP) - i.e., one concept is

\footnotetext{
${ }^{3}$ Mean Length of Utterance - a measure of language proficiency which is defined as ratio of the total number of morphemes by the total number of sentences
} 
VII Congresso Brasileiro de Informática na Educação (CBIE 2018)

Anais do XXIX Simpósio Brasileiro de Informática na Educação (SBIE 2018)

defined by one picture concept (cf. Figure 1-A); and (iii) Semantic Compaction (SC) i.e., one concept is defined by a short sequence of pictures (cf. Figure 1-B). On the one hand, only literate people with no cognitive impairments can use the Alphabet-Based systems. On the other hand, SMP and SC can be used for non-literate or people with cognitive impairments. The SMP is easier to understand and use, and provides immediate feedback with only one click. However, some abstract words (e.g., adjectives and prepositions) are difficult to represent with SMP. The SC allow the use of a wide range of meanings with fewer icons, however, this system is not obvious and requires training. Concerning the vocabulary organization, we can analyze it from users and system point of view. From the user's point of view, the vocabulary is organized based on (i) taxonomic representation (is- $a$ relations) or (ii) contextual disposition (situational relations). For example, in a taxonomic representation, Cup is organized under the Crokery class. In turn, in a contextual disposition, Cup can be organized into Kitchen or Restaurant contexts, for instance. From the system point of view, it is desirable that the internal vocabulary organization reflects user's mental lexicon (i.e., how the human mind organizes words), which has been done with (i) ontologies in Martínez-Santiago et al. (2015), and (ii) the frame-semantic approach in Hernández et al. (2014) to model the cognitive process underlying language use. An ontology is a taxonomy with a set of inference rules that allow automatic reasoning on its elements [Gruber 1995], while a semantic frame is a data structure that represents familiar objects and common situations [Lowe et al. 1997] - for example, the Ingestion frame model the verb to eat has two frame elements Ingestor and Ingestible. These two approaches take advantages of semantic databases as the FrameNet that implements the semantic-frame approach seen above and the WordNet ${ }^{4}$ that provides lexical and semantic relations between words.

(A)

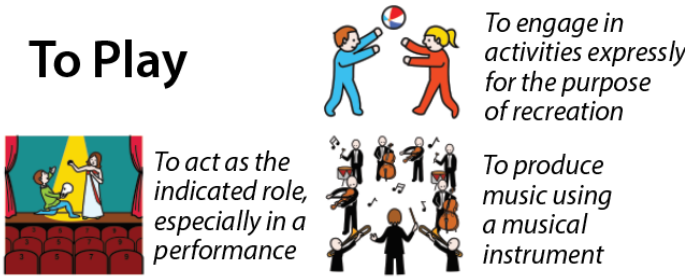

(B)
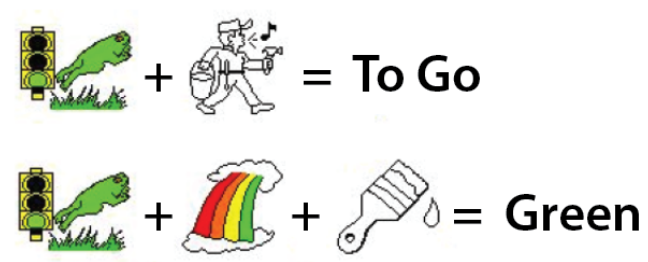

Figure 1. (A) Single Meaning Picture: three different ARASAAC pictograms (www.arasaac.org) for the concept To Play. (B) Semantic Compaction: multimeaning icons of Minspeak $®$ (www.minspeak.com) for the concepts go and green.

The interface customization is related with the accessibility and comprises visual, hearing and the manipulation settings of the AAC system. Concerning the visual settings, a robust AAC system must offer adaptations in the pictograms label size and font, as well as contrast and color scheme adaptations concerning user's visual ability (e.g., low vision and color blindness). Moreover, the system must allow the configuration of the number of pictograms per screen to reflect user's cognitive ability (e.g., perception, attention and memory). Hearing settings should reflect users' needs for volume, clarity, and sharpness according to problems as hearing loss or auditory processing disorder. Finally, regarding the manipulation, it must consider user's motor

\footnotetext{
${ }^{4}$ WordNet: a lexical database of English language - https://wordnet.princeton.edu/
} 
VII Congresso Brasileiro de Informática na Educação (CBIE 2018)

Anais do XXIX Simpósio Brasileiro de Informática na Educação (SBIE 2018)

ability (e.g., limbs movement, fine or gross motor skills). This way, AAC systems should provide alternative input methods such as switches, scanning, eye gaze, and brain-computer interface to be adapted to a large number of users.

\subsection{Communication}

Here we deal with four major aspects: pictogram selection, construction of meaningful sentences, flexibility, and feedback. The first is presented as follow, while the others are detailed in next paragraphs. The pictogram selection comprises (i) color-coding systems and (ii) Motor Planning. On the one hand, the color-coding systems are used to quickly find pictograms in a group by searching for colors that carry some meaning [Fitzgerald 1949], [Bryan 2008]. The most popular color coding system is the Fitzgerald Key [Fitzgerald 1949] which groups concepts into six colors regarding its grammatical role (cf. Figure 2-A) and was used to teach deaf children to read by structuring sentences correctly. The Colorful Semantics [Bryan 2008] group concepts into five color-coded incremental levels based on the meaning of words (i.e., semantics) (cf. Figure 2-B) and it is used to develop children's grammar. Notice that only the purple color (i.e., Describe) can be moved to different places in the sentence to add a description to other words (cf. Figure 2-C). On the other hand, the Motor Planning [Halloran and Emerson 2006] is related to the pictogram localization in the screen. The Motor Planning encompasses the planning and execution of a series of movements to select a specific pictogram, which brings consistency in pictogram search path and avoids variants in the same pictogram selection. These consistent motor patterns for pictogram selection allow the development of automaticity in communication. That is, the user learns the path (i.e., a sequence of clicks and swipes in the screen) to a specific pictogram and do not need to think about the pictogram path anymore.

(A)

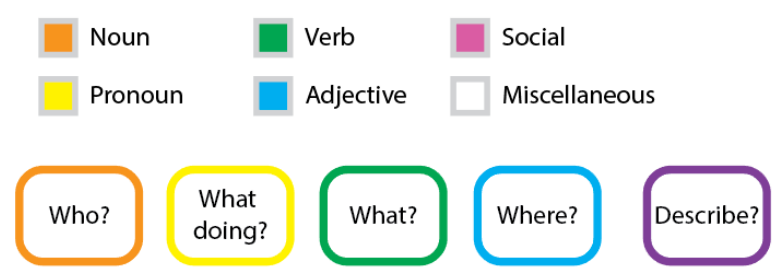

(C)

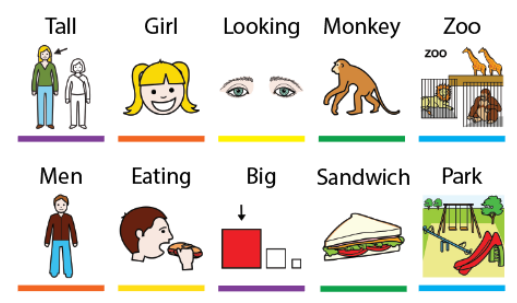

Figure 2. (A) Fitzgerald Key. (B) Colorful Semantics. (C) Telegraphic sentence using Colorful Semantics.

The construction of meaningful sentences comprises a variety of strategies that allow the construction of syntactically and semantically correct sentences. We will detail three strategies: (i) the theory of scripts [Todman et al. 2008], (ii) the prediction techniques, and (iii) the COMPANSION system [Demasco and McCoy 1992]. In the theory of scripts [Todman et al. 2008], a script denotes a structured communication dialog likely predictable. For example, a script can specify a sequence of steps required to complete a particular task (e.g., buy something in a grocery store). AAC developers can implement such theory using questions and answers, and the frame-semantic approach (cf. Section 3.1). This way, the communication is the result of the answers combination. The prediction techniques guide the user's communication by reducing the number of possible pictograms they have available at a given moment. More precisely, one can distinguish two specific kinds of prediction during sentence construction: when 
VII Congresso Brasileiro de Informática na Educação (CBIE 2018)

Anais do XXIX Simpósio Brasileiro de Informática na Educação (SBIE 2018)

the user selects the very first pictogram or when the user has already chosen one or more pictograms. In the first case, the system can suggest pictograms based on (i) context knowledge, (ii) user's log, or (iii) general statistical knowledge; in the second case, in addition to the previews points, the system can also suggest pictograms according to (iv) natural language aspects (i.e., syntax, semantics, and pragmatics) or (v) improved statistical knowledge considering previously selected pictograms. We will detail these points next. Contextual knowledge as time and geographical localization (e.g., School is a diary event that takes place between 8 am and noon when the user is near to the school) can be used to suggest contextual-related pictograms. User's log with previously built sentences can also be used as a start point in prediction. Concerning the general statistical knowledge, one can use large text corpora (e.g., CHILDES Corpus Database ${ }^{5}$ ) as a training dataset to extract the most used single words and the most used colocations (i.e., the habitual juxtaposition of two words). Regarding the natural language aspect, the syntax deals with the arrangement of pictograms to create well-formed sentences in a language (e.g., English or Portuguese), the semantics deals with the meaning of the sentence, and the pragmatics deals with the contexts in which the language is used. To address natural language aspects, one can use simple grammatical rules based on colorcode schemes (i.e., Fitzgerald Key and Colorful Semantics) and semantic-frames. The first one can be used as a clue for the user to identify missing pictograms in the sentence, while the second one can establish the correct complement for a given verb (e.g., the verb eat requires an eatable thing as a complement). Indeed, empirical evidence found in [Bolderson et al. 2011] show that the adoption of Colorful Semantics yielded good results for children with severe communicative disorders since they often omit verbs and grammatical elements and fail to build complete sentences. One can also use semantic databases (e.g., FrameNet and WordNet) to add semantic and pragmatic restrictions on the simple grammar described above. Finally, concerning the improved statistical knowledge, it is based on the general statistical knowledge improved by restrictions established by previews selected pictograms. We highlight that the theory of scripts can also be regarded as a prediction technique since a given script can be employed to implement a conversational recommender [Hernández et al. 2014] that makes suggestions and refinements based on the user's feedbacks. After the sentence construction, the COMPANSION system [Demasco and McCoy 1992] can be used to expand the telegraphic language (i.e., the compressed message made by sentences with missing words - "Daddy here") into the full and grammatically corrected sentence (i.e., "Daddy is here").

The flexibility comprises two aspects [Hill 2006]: (i) freedom of communication and (ii) shortcuts to speed up the communication. The first one deals with Novel Utterance Generation (SNUG), which means that the user can communicate whatever they want by combining different pictograms, including the possibility to insert pictograms in any part of the sentence - not only at the end -, and change the pictogram position in the sentence. The second one deals with strategies to make faster the communication, including pictogram prediction for suggesting candidate pictograms, and Pre-Programmed Utterances (PPU) with full sentences/expressions (e.g., "I would

${ }^{5}$ Child Language Data Exchange System - http://childes.psy.cmu.edu/ 
VII Congresso Brasileiro de Informática na Educação (CBIE 2018)

Anais do XXIX Simpósio Brasileiro de Informática na Educação (SBIE 2018)

like to tell you something", "I feel better!", and "Hello!"), and phrase templates (e.g., "I would like to..." and "Can you...") to be completed with other pictograms.

The feedback during the communication aids the user to understand the system status. It comprises two aspects, the visual and the sound one. The user must receive an appropriate and rapid feedback for each action performed in the system, including: (i) a sonorous reaction of each click in the interface elements and (ii) a sentence construction area for grouping the selected pictograms.

\section{Reference Architecture for AAC Systems}

Figure 3 shows the RA for instantiating concrete architectures for AAC systems. This RA is segmented into three layers: data, logic, and interface. These layers are responsible respectively for the data storage, business rules, and user's interaction. Considering that we built this RA to encompass all the requirements of a robust AAC system shown in Section 3, we have components to deal with each requirement. Next, we show the components of each layer, and how the RA connect them.

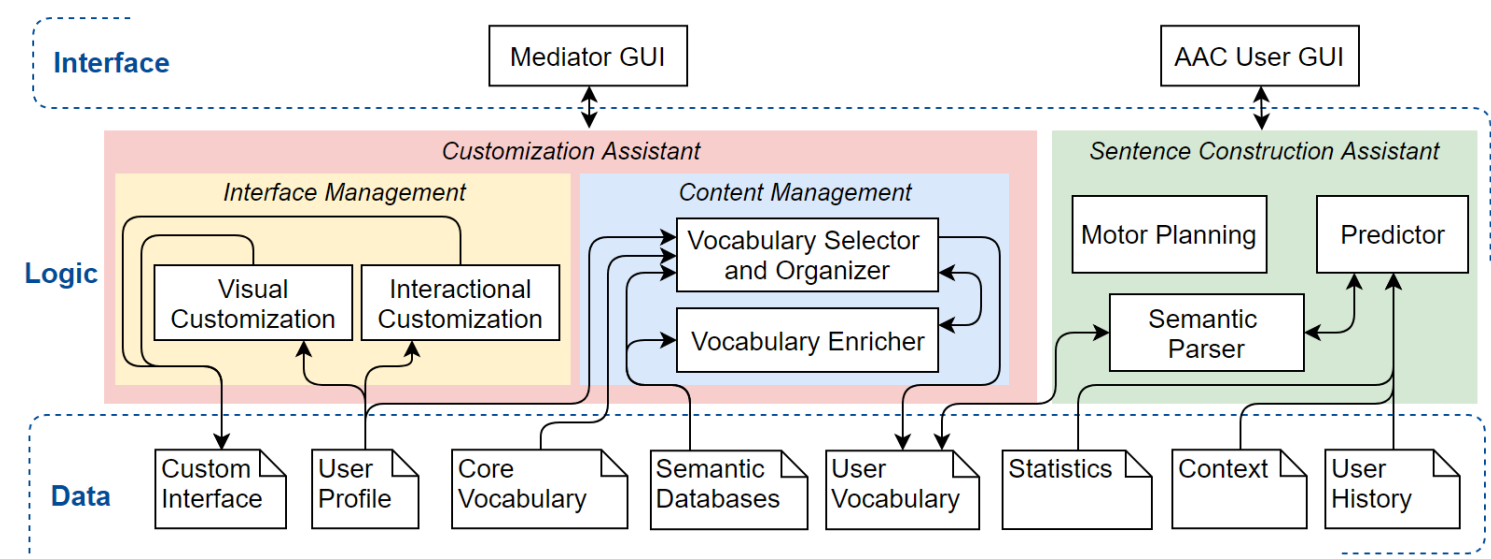

Figure 3. Reference Architecture for AAC Systems.

\subsection{Data Layer Components}

The data layer is composed of eight components detailed next. The User Profile encompasses characteristics (e.g., communicational development, cognitive, motor and visual ability) and preferences (e.g., size and quantity of pictograms per screen and color-coding system) that can make the AAC system fit better for user needs. In turn, the Custom Interface is the AAC system interface after the customization process over the User Profile. The Core Vocabulary corresponds to core words organized into a set of core categories (e.g., taxonomic or contextual). In this approach, the core words bring instances while the core categories bring important concepts/themes for communication. This way, the user can start with the default core words and then, based on core categories, can improve and customize the User Vocabulary based on recently learned words. Semantic Databases encompasses lexical and semantic repositories such as WordNet, FrameNet, which are used to provide connections between words in the User Vocabulary. Finally, the Statistics encompasses information about common words and colocations, the Context corresponds to rules that relate the use of pictograms set to variables like time and geographical localization, and User History encompasses the system log with previous build sentences of each AAC user. 
VII Congresso Brasileiro de Informática na Educação (CBIE 2018)

Anais do XXIX Simpósio Brasileiro de Informática na Educação (SBIE 2018)

\subsection{Logic Layer Components}

This layer is composed of two main modules named (i) Customization Assistant and (ii) Sentence Construction Assistant. The first is presented as follow, while the second is detailed in next paragraph. The Customization Assistant manages the graphical components and the content of the AAC User GUI. It is subdivided into two modules: Interface Management and Content Management. The first module comprises two components: the Visual Customization and the Interactional Customization. These components consume information from User Profile to customize, respectively, the way users see the interface (e.g., color, size, and quantity of pictograms per screen) and the way users interact with the interface (e.g., by means of semantic-frame completion, questions and answers or freely interaction as well as the output method). The second module comprises two components: Vocabulary Selector and Organizer and Vocabulary Enricher. The Vocabulary Selector and Organizer are responsible for taking the Core Vocabulary, the User Profile and the Semantic Databases to organize the concepts into taxonomic or contextual categories, and to establish syntactic and semantic connections among concepts to generate the User Vocabulary. We highlight that the Core Vocabulary is only a baseline that makes easier the initial AAC vocabulary usage, and new words can also be added using the Vocabulary Enricher. This component receives the new word and, using semantic similarity and sense disambiguation techniques [Wei et al. 2015] with concepts from Semantic Databases and User Vocabulary, has evidence that helps to decide the best place in the organization to add the new word.

The Sentence Construction Assistant is responsible for guiding the user in the sentence construction task. It has three components: Motor Planning, Semantic Parser, and Predictor. The Motor Planning allows the easy search of pictograms by keeping each pictogram always on the same screen area (e.g., persons and pronouns always appear on the left side). The Semantic Parser component is responsible for verifying the syntactic and semantic correctness of the build in sentences. For doing this, this component has a simplified grammar for the syntactic verification and needs to access the semantic relations in the User Vocabulary for the semantic verification. The Semantic Parser also deals with the expansion of telegraphic sentences (i.e., the COMPANSION technique) to its full and grammatically corrected form. In turn, the Predictor component uses information from Statistics, Context and User History data plus the Semantic Parser output to suggest pictograms for the construction of meaningful sentences.

\subsection{Interface Layer Components}

This layer is composed of two components, the Mediator GUI and the AAC User GUI. The Mediator GUI is used by the mediator (i.e., health and education professionals or user's relatives) for customizing the content (i.e., vocabulary and exhibition) of the AAC system according to the User Profile. In turn, the AAC User GUI is used by disabled individuals in communicating with others. Notice that developers can implement these two GUI as two views of the same application or two different applications. 
VII Congresso Brasileiro de Informática na Educação (CBIE 2018)

Anais do XXIX Simpósio Brasileiro de Informática na Educação (SBIE 2018)

\section{Conclusion}

In this paper, we discussed the major requirements that a robust AAC system must have and presented our proposal for a Reference Architecture (RA). This RA is conceptual and technology-independent. This way, developers can see this RA as a baseline for instantiating and evaluate concrete software architectures for several goals such as introducing the symbolic communication, improve language skills, and facilitate the literacy process, to name a few.

Nowadays we are using this architecture for refactoring the architecture of aBoard [Lima et al. 2017], [Franco et al. 2017] - a mobile and cloud-based platform for $\mathrm{AAC}$ and inclusive education. Moreover, the Core Vocabulary with core words and core categories is in progress. For this, we are performing Natural Language Processing analysis over children utterances of CHILDES Corpus Database, and semantic data from WordNet and FrameNet. Future work concerns the evaluation of the refactored version of aBoard regarding the following tasks: pictogram navigation and prediction, as well as the construction of meaningful sentences. Such evaluations will be conducted to assess the effectiveness of the instantiated RA according to the five major requirements we have discussed in this paper: 1) Vocabulary customization; 2) Interface customization; 3) Communication flexibility; 4) Appropriate feedback; and 5) Construction of meaningful sentences.

\section{Acknowledgments}

This research was supported by the National Center for Scientific and Technological Development (CNPq) [458798/2013-4 and 461492/2014-8] and, the Coordination of Improvement of Higher Education Personnel (CAPES) [88887.095638/2015-01].

\section{References}

Balandin, S. \& Iacono, T. (1999), "Crews, Wusses, and Whoppas: core and fringe vocabularies of Australian meal-break conversations in the workplace", Augmentative and Alternative Communication, v.15, n.2.

Bolderson, S., Dosanjh, C., Milligan, C., Pring, T. \& Chiat, S. (2011), "Colourful Semantics: A clinical investigation”. Child Language Teaching and Therapy, 27(3).

Bryan, A. (2008), "Colourful Semantics: Thematic Role Therapy", Language Disorders in Children and Adults, Whurr Publishers Ltd.

Cloutier, R., Muller, G., Verma, D., et al. (2010). "The concept of reference architectures". Systems Engineering, v.13, n.1.

Demasco, P. W. \& McCoy, K. F. (1992), "Generating text from compressed input: an intelligent interface for people with severe motor impairments", Communications of the $A C M$, v.35, n.5.

Fitzgerald, E. (1949), "Straight Language for the Deaf. A System of Instruction for Deaf Children", Alexander Graham Bell Association for the Deaf.

Franco, N. M., Lima, A. L., Lima, T. P., Silva, E. A., Lima, R. J. \& Fidalgo, R. N. (2017), “A Recall Analysis of Core Word Lists over Children's Utterances for Augmentative 
VII Congresso Brasileiro de Informática na Educação (CBIE 2018)

Anais do XXIX Simpósio Brasileiro de Informática na Educação (SBIE 2018)

and Alternative Communication", In International Symposium on Computer-Based Medical Systems (CBMS).

Franco, N., Lima, T., Lima, A., Silva, E., Lima, R., Cavalcante, T., \& Fidalgo, R. (2017). "aBoard: Uma Plataforma para Educação Inclusiva a partir de Comunicação Aumentativa e/ou Alternativa". In Simpósio Brasileiro de Informática na Educação, v.28, n.1.

Gruber, T. R. (1995), "Toward principles for the design of ontologies used for knowledge sharing". International journal of human-computer studies, 43(5-6).

Halloran, J. \& Emerson, M. (2006), "LAMP: Language acquisition through Motor Planning", Wooster (OH): Prentke Romich Company.

Hernández, S. S., Mancilla, D., Medina, J. M. \& Iregui, M. (2014), “User-centric Recommendation Model for AAC based on Multi-criteria Planning", In International Conference on Advances in Human-oriented and Personalized Mechanisms, Technologies and Services.

Hill, K. (2006). "Augmentative and alternative communication (AAC) research and development: the challenge of evidence-based practice", International Journal of Computer Processing of Oriental Languages, 19.04.

Kagohara, D., Van der Meer, L., Ramdoss, S., O’ Reilly, M. F., Lancioni, G. E., Davis, T. N., Rispoli, M., Lang, R., Marschik, P., Sutherland, D., Green, V., Sigafoos, J. (2013). "Using iPods and iPads in teaching programs for individuals with developmental disabilities: A systematic review", Research in Developmental Disabilities, 34.

Lima, T., Silva, E., Lima, A., Franco, N., \& Fidalgo, R. (2017), “aBoard: uma Plataforma Computacional na Nuvem para Comunicação Alternativa e Educação Inclusiva”. In Anais dos Workshops do Congresso Brasileiro de Informática na Educação, v.6, n. 1.

Lowe, J. B., Baker, C. F. \& Fillmore, C. J. (1997), "A frame-semantic approach to semantic annotation", Proceedings 1997 Siglex Workshop/ANLP97.

Martínez-Santiago, F., Díaz-Galiano, M. C., Ureña-López, L. A. \& Mitkov, R. (2015), “A semantic grammar for beginning communicators", Knowledge-Based Systems, v.86.

McNaughton, D., \& Light, J. (2013). "The iPad and mobile technology revolution: Benefits and challenges for individuals who require augmentative and alternative communication", Augmentative and Alternative Communication, 29:2.

Newell, A., Langer, S. \& Hickey M. (1998), "The rôle of natural language processing in alternative and augmentative communication", Natural Language Engineering, v.4, n. 1.

Pino, A. \& Kouroupetroglou, G. (2010), "ITHACA: An Open Source Framework for Building Component-Based Augmentative and Alternative Communication Applications", ACM Transactions on Accessible Computing, v.2, n.4.

Todman, J., Alm, N., Higginbotham, J. \& File, P. (2008), "Whole utterance approaches in AAC", Augmentative and Alternative Communication, v. 24, n.3.

Wei, T., Lu, Y., Chang, H., Zhou, Q. \& Bao, X. (2015), "A semantic approach for text clustering using WordNet and lexical chains", Expert Systems with Applications, v.42, n.4. 